\title{
Polyphenols in human nutrition: from the in vitro antioxidant capacity to the beneficial effects on cardiometabolic health and related inter-individual variability - an overview and perspective
}

\author{
T. Ruskovska ${ }^{1 *}$, V. Maksimova ${ }^{1}$ and D. Milenkovic ${ }^{2,3}$ \\ ${ }^{1}$ Faculty of Medical Sciences, University 'Goce Delcev', 2000 Stip, North Macedonia \\ ${ }^{2}$ Department of Internal Medicine, Division of Cardiovascular Medicine, School of Medicine, University of California Davis, \\ Davis, CA 95616, USA \\ ${ }^{3}$ Université Clermont Auvergne, INRA, UNH, CRNH Auvergne, F-63000 Clermont-Ferrand, France
}

(Submitted 6 April 2019 - Final revision received 2 October 2019 - Accepted 14 October 2019 - First published online 29 October 2019)

\section{Abstract}

Oxidative damage of cells and tissues is broadly implicated in human pathophysiology, including cardiometabolic diseases. Polyphenols, as important constituents of the human diet and potent in vitro free radical scavengers, have been extensively studied for their beneficial effects on cardiometabolic health. However, it has been demonstrated that the in vivo antioxidant activity of polyphenols is distinct from their in vitro free radical-scavenging capacity. Indeed, bioavailability of nutritional polyphenols is low and conditioned by complex mechanisms of absorption, distribution, metabolism and excretion. Nowadays, it is commonly accepted that the cellular antioxidant activity of polyphenols is mainly carried out via modification of transcription of genes involved in antioxidant defence. Importantly, polyphenols also contribute to cardiometabolic health by modulation of a plethora of cellular processes that are not directly associated with antioxidant enzymes, through nutri(epi)genomic mechanisms. Numerous human intervention studies have demonstrated beneficial effects of polyphenols on the key cardiometabolic risk factors. However, inconsistency of the results of some studies led to identification of the inter-individual variability in response to consumption of polyphenols. In perspective, a detailed investigation of the determinants of this inter-individual variability will potentially lead us towards personalised dietary recommendations. The phenomenon of inter-individual variability is also of relevance for supplementation with antioxidant (pro)vitamins.

\section{Key words: Polyphenols: Antioxidants: Cell signalling: Nutrigenomics: Cardiometabolic health}

Nutrition is essential for the maintenance of optimal health. Both under- and overnutrition lead to disease development and increase in the rate of mortality. Both phenomena are still present in human society, and it is rather difficult to overcome them, despite the unprecedented technological advances in food science in the past few decades. In addition, we witness the occurrence of obesity accompanied by deficit of essential nutrients and plant food bioactives in low- and middle-income countries, as well as in low-income subgroups in developed countries $^{(1,2)}$. Other than economic status, it has also been suggested that the educational status has a significant influence on food choices ${ }^{(3)}$.

Plant food bioactives are important constituents of the human diet. Although not classified as essential nutrients, they may have beneficial effects on human health. Unlike vitamins, their inadequate intake will not result in specific syndrome of deficit, but rather in increased risk of chronic diseases. The plant food bioactives include terpenoids (isoprenoids), phenolic compounds (including polyphenols), glucosinolates, betalains and others. In this review, we will focus on polyphenols and their effects on human cardiometabolic health. Indeed, there is growing evidence about the preventive effects of polyphenols on cardiometabolic diseases, such as type 2 diabetes mellitus ${ }^{(4)}$ and atherosclerosis ${ }^{(5)}$. There is also evidence that polyphenols exert beneficial effects on neurodegenerative diseases $^{(6)}$ and some forms of cancer $^{(7)}$, which will not be addressed on this occasion.

Because of their remarkable in vitro free radical-scavenging properties, early investigators attributed the beneficial effects of polyphenols on human health exclusively to their capacity to

Abbreviations: AKT, serine/threonine kinase; Keap1, Kelch like ECH associated protein 1; Nrf2, nuclear factor, erythroid 2 like 2; RCT, randomised controlled trial; ROS, reactive oxygen species.

* Corresponding author: T. Ruskovska, email tatjana.ruskovska@ugd.edu.mk 
scavenge free radicals ${ }^{(8,9)}$. Regarding their cardioprotective effects, many studies focused on LDL oxidation, which plays central role in atherogenesis ${ }^{(10)}$. Indeed, it has been demonstrated that upon consumption of foods and beverages rich in polyphenols, the resistance of LDL to oxidation increases ${ }^{(11)}$. Notably, LDL oxidisability is still of interest ${ }^{(12)}$. Following these observations, polyphenols became an integral part of the theory of oxidative stress.

Therefore, in the first part of this review, we will give an overview of the theory of oxidative stress and a summary of the results of antioxidant supplementation studies. In the second part, we will focus on polyphenols and their antioxidant properties, which will be followed by an overview of their effects on cardiometabolic health, and our present understanding of the inter-individual variability in the response of their consumption.

\section{Oxidative stress and antioxidant supplementation}

\section{Definition of oxidative stress and mechanism of lipid} peroxidation

The term 'free radical' refers to the chemical species containing one or more unpaired electrons, which encompass various $\mathrm{O}-, \mathrm{C}-$, N- or S-centred radicals. Among them, the O-centred radicals are the most prevalent in the biological systems ${ }^{(13)}$, including the superoxide radical and the highly reactive hydroxyl radical. These, along with the non-radical hydrogen peroxide, are the most common reactive oxygen species (ROS). Following Harman's free radical theory of aging (1956) ${ }^{(14)}$, the term 'oxidative stress' was first mentioned in 1970, referring to the oxidative challenge of erythrocytes by hydrogen peroxide $^{(15)}$. In 1985, oxidative stress was defined as an imbalance between oxidants and antioxidants, in favour of the former ${ }^{(16)}$, focusing on the oxidative damage of cells, tissues and organs ${ }^{(17)}$. Since then, the concept has evolved substantially, leading to redefinition of oxidative stress as a dysfunction of cellular redox signalling and redox control ${ }^{(18,19)}$. Most recently, a distinction has been made between oxidative eustress, as a physiological condition that is essential for redox signalling, and oxidative distress, as a supraphysiological condition that causes damage to biomolecules ${ }^{(20)}$. So far, hundreds of thousands of papers have been published demonstrating and confirming the association of oxidative stress with various human diseases, including cardiometabolic ones. Indeed, nowadays, it is almost impossible to find a human disease or condition that is not associated with the oxidative stress.

Classical theory describes the oxidative stress as an overall imbalance between the ROS from one side and the cellular antioxidants from the other, of course in favour of ROS. This imbalance occurs because of an excessive production of ROS, or an inadequate concentration and/or activity of the cellular antioxidants (non-enzymatic or enzymatic), or because of both $^{(21)}$. The classical understanding of the oxidative stress was a leitmotif of the early attempts to translate the immense amount of scientific evidence into clinical practice. Within these efforts, it seems that the chain reaction of lipid peroxidation attracted most attention. Hydroxyl radical (HO·), as the most reactive ROS, reacts in a high rate reaction with PUFA, in the first place linoleic or arachidonic acid of membrane phospholipids. Hydroxyl radical abstracts hydrogen atom from PUFA, which starts the autocatalytic chain reaction of lipid peroxidation. In this initiating reaction, a carbon-centred lipid radical is formed $(\mathrm{L} \cdot)$, which further reacts with molecular oxygen to generate an oxygen-centred lipid peroxyl radical (LOO.). The lipid peroxyl radical can attack another PUFA to generate lipid hydroperoxide (LOOH) and a new carbon-centred lipid radical, which propagates the chain reaction ${ }^{(22)}$. Lipid hydroperoxides have the ability to react with trace metals to generate lipid alkoxyl radicals (LO·). Both lipid peroxyl radicals and lipid alkoxyl radicals undergo cyclisation and/or degradation, generating a plethora of reactive aldehydes such as malondialdehyde (MDA), trans-4-hydroxy-2-nonenal, trans-4-oxo-2-nonenal, acrolein, glyoxal and many others. What reactive aldehydes will be generated depends on the type of PUFA subjected to lipid peroxidation $^{(23)}$. Importantly, these reactive aldehydes have the ability to oxidise proteins ${ }^{(24)}$ and $\mathrm{DNA}^{(25)}$. Therefore, if present in excess and/or for a longer period, they can induce cellular damage and consequently induce disease development.

Lipid peroxyl radicals can be scavenged not only by vitamin $\mathrm{E}$ but also by other nutrients such as polyphenol or $\beta$-carotene ${ }^{(26)}$, which terminates the chain reaction of lipid peroxidation. Notably, the chemistry behind the scavenging of lipid peroxyl radicals by polyphenols and vitamin $\mathrm{E}$ is very similar ${ }^{(27)}$. Following the reaction of termination by vitamin E, a relatively stable $\alpha$-tocopheryl radical is formed ( $\alpha$-tocopherol is the predominant form of vitamin $\mathrm{E}$ in human tissues $\left.{ }^{(28)}\right)$. The $\alpha$-tocopheryl radical can be oxidised to a stable non-radical quinone or reduced back (recycled) to hydroquinone by vitamin $\mathrm{C}^{(29)}$. However, the biological relevance of the later reaction is not completely clear ${ }^{(30)}$. Other possibilities for termination of the chain reaction of lipid peroxidation are the following: substrate unavailability, collision of two LOO. molecules or reaction of LOO. with other radicals.

\section{Antioxidant supplementation studies - hopes and pitfalls}

Undoubtedly, if present at high concentrations, ROS, free radicals and reactive aldehydes will cause cellular damage and eventually disease. Therefore, for early investigators, it seemed logical that supplementation with low molecular weight antioxidants would prevent the cellular damage, resulting in beneficial effects on human health and decreased mortality. Following this assumption, numerous randomised controlled trials (RCT) have been conducted with the aim to study the effects of supplementation with vitamin $\mathrm{C}$, vitamin $\mathrm{E}, \boldsymbol{\beta}$-carotene, vitamin $\mathrm{A}$ and $\mathrm{Se}$, alone or in various combinations, on oxidative stress and health maintenance. Some of these studies included patients with various chronic diseases. In others, the relevance of antioxidant supplementation in primary prevention was studied on healthy subjects. A meta-analysis of sixty-seven RCT was conducted and published in $2008^{(31)}$, the results of which were discouraging. The same authors repeated the meta-analysis, including the papers that were published subsequently and the updated meta-analysis of seventy-eight RCT was published in $2012^{(32)}$. The results were similar, that 
is, showing 'no evidence to support health effects of supplementation with low molecular weight antioxidants in both primary and secondary prevention'. Moreover, it has been demonstrated that supplementation with vitamin $\mathrm{E}, \beta$-carotene and vitamin $\mathrm{A}$ may slightly increase mortality. After the initial disappointment, a quest for rational explanation has been initiated and several important issues have been raised, which will be presented in the following paragraphs.

Over-simplification of the theory of oxidative stress has been identified as an important cause of the disappointing results of the antioxidant supplementation studies, which were an attempt to translate the accumulated scientific evidence into practice. This over-simplification has been referred as a translational shortcut'(33). Indeed, behind the simplified representation of the oxidative stress as an overall imbalance between ROS and antioxidants, there is a complex network of cellular redox regulators. These include antioxidant enzymes and low molecular weight antioxidants that work together, and along with other cellular factors, to establish and maintain the cellular redox balance. Obviously, an over-simplification of this complex network down to unselective supplementation with high doses of free radical scavengers was not effective. Moreover, the finding of increased mortality points out that 'natural', which refers to both vitamin $\mathrm{E}$ and $\beta$-carotene, is not equal to 'safe in high doses'. Another potential issue might be the composition and/or quality of supplements, which would be extremely difficult to address from our perspective.

In the antioxidant supplementation studies, an important factor that should have been taken into account is the role of reactive aldehydes as second messengers of free radicals. Indeed, there is evidence that, in its physiological concentrations, trans-4-hydroxy-2-nonenal acts as a signalling molecule, and as such is involved in many biological processes ${ }^{(34)}$, including adaptive response to other oxidative stressors ${ }^{(35)}$. Therefore, supplementation with high doses of vitamin E may impair the physiological role of trans-4-hydroxy-2-nonenal, potentially leading to adverse effects. It is indicative that an increased mortality was observed in supplementation with vitamin $\mathrm{E}$, $\beta$-carotene and vitamin $\mathrm{A}$, all of them liposoluble compounds, but not with vitamin C. Indeed, liposolubility aggravates elimination, which may be an important element for development and manifestation of adverse effects.

Vitamin $\mathrm{E}^{(36)}$ and $\beta$-carotene ${ }^{(37)}$ have multiple biological functions. Currently, it is not completely understood how these functions were implicated in the results of the RCT of antioxidant supplementation. However, some aspects have been discussed in a recent review ${ }^{(38)}$. Namely, it has been demonstrated that $\beta$-carotene inhibits the enzymatic activity of glutathione-Stransferase $\pi$, which provides protection against benzo(a)pyrene diol epoxide, a toxic metabolite of tobacco smoke. The decreased enzymatic activity of glutathione-S-transferase $\pi$ has been proposed as the underlying mechanism of the increased incidence of lung cancer in smokers supplemented with $\beta$-carotene ${ }^{(39)}$. On the other hand, it has been demonstrated that vitamin E supplementation has beneficial health effects in specific groups of patients ${ }^{(40)}$, such as patients with nonalcoholic steatohepatitis ${ }^{(41)}$, diabetic patients with haptoglobin 2-2 genotype ${ }^{(42)}$, patients on haemodialysis ${ }^{(43)}$ and patients with oxidative stress-induced hypertension ${ }^{(44)}$. Moreover, some meta-analyses did not confirm the finding regarding the increased mortality in persons supplemented with vitamin $\mathrm{E}^{(45,46)}$. These examples illustrate the need to identify population groups and individuals who will most benefit from supplementation with antioxidant vitamins, as precisely as possible, including the dose and duration of supplementation. To the best of our knowledge, the inter-individual variability of response to low molecular weight antioxidants has not been explored in detail so far. Biomarkers of oxidative stress ${ }^{(47)}$ shall be used for identification of patients who will most benefit from supplementation with antioxidant vitamins. However, the shortcomings of the currently available biomarkers ${ }^{(48)}$ do not allow their effective use in clinic. Therefore, a development of better, biologically much more specific and analytically more precise biomarkers of oxidative stress is of utmost importance.

\section{Polyphenols and their antioxidant properties}

\section{Polyphenols in the human diet}

Polyphenols are plant food bioactives that possess remarkable in vitro antioxidant capacity ${ }^{(49)}$. They have the ability to scavenge lipid peroxyl radicals ${ }^{(50)}$. As such, they are an integral part of the theory of oxidative stress ${ }^{(51)}$. Polyphenols are present at relatively high concentrations in the human diet. However, depending on individual ${ }^{(52)}$ and national nutrition habits, there is a large difference in daily consumption, ranging from 378 (SE 15) ${ }^{(53)}$ to 1757 (sD 696) ${ }^{(54)} \mathrm{mg} / \mathrm{d}$. Dietary polyphenols originate mainly from plant-based foods including fruits (mainly berries and apples), vegetables (onions, spinach, asparagus and broccoli), spices (black pepper, curcuma and saffron), whole grains, coffee, cocoa, tea, olive and various kinds of nuts ${ }^{(55)}$. Many of them are also administered as food supplements or herbal remedies. Polyphenols are well studied and classified. They are widespread in the plants, encompassing over 8000 different compounds ${ }^{(56)}$. However, the number of polyphenols that are relevant to human nutrition is much smaller. Two databases containing comprehensive information on nutritional polyphenols have been developed and are freely accessible: http://phenol-explorer.eu/ ${ }^{(57)}$ and https://www.ars.usda.gov/ ARSUserFiles/80400525/Data/Flav/Flav_R03-1.pdf (58).

Regarding the health benefits of consumption of foods and beverages rich in polyphenols, the first epidemiological study demonstrating an inverse association between flavonoid intake and mortality from CHD was published in $1993^{(59)}$. Unfortunately, another translational shortcut was taken here. Since polyphenols demonstrate high in vitro antioxidant capacity, it was assumed that the in vitro antioxidant capacity of any food or plant extract can be used as a direct determinant (or, in other words, a measure) of their beneficial health effects in humans. Following this concept, a list of in vitro antioxidant capacities of foods and beverages (measured with Oxygen Radical Absorbance Capacity assay) was published ${ }^{(60)}$. Besides the Oxygen Radical Absorbance Capacity assay ${ }^{(61,62)}$, several other methods were also developed, such as trolox equivalent antioxidant capacity, 2,2-diphenyl-1-picrylhydrazyl and ferric reducing antioxidant power assay ${ }^{(63)}$. However, 
growing scientific evidence started to elucidate some of the numerous and complex mechanisms of absorption, distribution, metabolism and excretion (ADME) of polyphenols, as molecular basis of their low bioavailability. It also became clear that the in vivo antioxidant activity of polyphenols involves much more complex mechanisms than the free radical scavenging and that molecular mechanisms that are not directly associated with the antioxidant enzymes are also involved in their health promoting effects. At the same time, the Oxygen Radical Absorbance Capacity list was misused by food and dietary supplement manufacturing companies to promote their products ${ }^{(64)}$. All this together led to withdrawal of the Oxygen Radical Absorbance Capacity list.

\section{Metabolism of nutritional polyphenols in the human body}

Bioavailability of nutritional polyphenols is low and far from uniform. Their plasma concentrations are at range of nм to low $\mu \mathrm{M}^{(65)}$. A notable exception is the gastrointestinal tract ${ }^{(66)}$, where the intestinal cells are in contact with high concentrations of polyphenols and other plant food bioactives. In the human body, polyphenols are subjected to complex and extensive metabolic transformations ${ }^{(67)}$, which significantly alter their chemical properties and sometimes diminish their antioxidant activity $^{(68)}$. Notably, lack of association between the antioxidant activity and some of the biological effects of polyphenol metabolites has been clearly observed ${ }^{(69)}$. Therefore, the in vitro total antioxidant capacity of foods, beverages or plant extracts cannot be a direct determinant of their biological effects.

Polyphenols are a heterogeneous family of plant food bioactives, encompassing several classes of bioactive compounds, such as flavonoids, phenolic acids, lignans, stilbenes and other polyphenols ${ }^{(57)}$. Because of the heterogeneity in their chemical structures, the metabolism of compounds that belong to different classes and subclasses, or even the metabolism of individual compounds, is different, which has been extensively reviewed elsewhere ${ }^{(67,70)}$. Here, we will give only a general overview of the metabolism of polyphenols, pinpointing the factors that are relevant for the inter-individual variability in response to their consumption. In the human diet, most of the polyphenols are found as glycosides. Hydrolysis of glycoside conjugates is a critical step in absorption of polyphenols in the small intestine, following which corresponding aglycones appear in the intestinal cells. In the intestinal cells, these aglycones are further subjected to an initial phase II metabolism, resulting in appearance of sulphate, glucuronide or methyl metabolites. These metabolites enter the circulation and reach the liver, where their phase II metabolism continues; only a small amount is transported back to the lumen of the small intestine, eventually reaching the large intestine. Therefore, after the absorption in the small intestine, polyphenol phase II metabolites are predominantly found in the circulation $^{(71)}$, whereas the appearance of parent compounds, such as (-)-epicatechin-3-O-gallate and (-)-epigallocatechin-3O-gallate, is practically an exception of the general rule. Post-absorption plasma concentrations of phase II metabolites are low, reflecting the low bioavailability of polyphenols, which is further emphasised by their rapid urinary excretion. A plethora of enzymes and transporters is involved in the processes of hydrolysis, absorption, phase II metabolism and renal excretion of polyphenols ${ }^{(67)}$. Theoretically, polymorphisms in the genes that code these proteins may be involved in the absorption, distribution, metabolism and excretion component of the overall inter-individual variability in response to consumption of nutritional polyphenols. Indeed, it has been demonstrated that catechol-O-methyltransferase (enzyme that catalyses methylation of nutritional polyphenols in the human body) genotype (rs4680) may determine the health benefits from green tea intake ${ }^{(72)}$. However, the existing data are limited, and genetic factors, along with the influence of gut microbiota, should be addressed in more detail in future studies $^{(73)}$.

Indeed, only a small portion of the nutritional polyphenols is absorbed in the small intestine, while most of them reach the large intestine where they are subjected to extensive metabolism. Gut microbiota plays an important role in the metabolism of nutritional polyphenols, which results in the appearance of a plethora of gut microbiota metabolites, such as aglycones, small phenolic acids and valerolactones. Some of these molecules are absorbed in the large intestine and are further metabolised in the liver. There is strong scientific evidence that gut microbiota metabolites are among the key mediators of the (cardiometabolic) health promoting effects of polyphenols ${ }^{(74,75)}$. In addition, with identification of different metabotypes ${ }^{(76,77)}$, gut microbiota has been pinpointed as one of the determinants of inter-individual variability in response to consumption of polyphenols.

\section{Molecular mechanisms involved in the antioxidant effects of polyphenols and beyond}

With the evolution of our understanding of oxidative stress (see section 'Definition of oxidative stress and mechanism of lipid peroxidation') and elucidation of the complex metabolism of polyphenols in the human body (section 'Metabolism of nutritional polyphenols in the human body'), the concept that health promoting effects of polyphenols are mediated solely by their ability to scavenge free radicals in vivo has been abandoned. Indeed, it has been clarified that polyphenol metabolites do not scavenge substantial amount of free radicals in vivo and that $\alpha$-tocopherol is the key compound that terminates the chain reaction of lipid peroxidation. Hence, summarising the latest scientific evidence from both fields, the major mechanism by which polyphenols contribute to the cellular antioxidant defence has been proposed recently ${ }^{(78)}$. Namely, after consumption, low concentrations of mainly metabolised polyphenols reach the cells. Some of them, such as catechin, epicatechin, hydroxytyrosol, delphinidin and carnosic acid, are oxidised by free radicals, in free radical-scavenging reactions. An important target of the oxidised polyphenols is Keap1 (Kelch like ECH associated protein 1), a cytosolic protein whose function is to regulate the activity of transcription factor Nrf2 (nuclear factor, erythroid 2 like 2). Some polyphenols, such as resveratrol and curcumin, bind with 
Keap1 directly, without prior oxidation. The regulatory function of Keap1 consists of assistance in ubiquitination of Nrf2, which results in its degradation by proteasome. After binding a polyphenolic compound, Keap1 is inactivated and the halflife of Nrf2 is extended. Therefore, Nrf2 migrates to the nucleus, binds to the antioxidant response element (also known as electrophile response element) and initiates the transcription of genes that are involved in the antioxidant defence and detoxification. Besides Nrf2, other transcription factors, such as c-Maf, c-Jun, c-Fos, Fra1, Bach1, Nrf1 or c-Myc, are also involved in the antioxidant response element-mediated transcription in a complex interplay. In addition, there is evidence that protein kinases, such as protein kinase $\mathrm{C}$ or phosphoinositide-3-kinase, play role in the phosphorylation of $\mathrm{Nrf} 2$, which is a critical step for its translocation to the nucleus. Recent findings that polyphenol metabolites have potential to modulate kinases' activities are indicative of multilevel regulation of Nrf2mediated transcription.

Besides the Keap1/Nrf2/antioxidant response element pathway, nutritional polyphenols also modulate a plethora of cellular functions that are not directly connected with the enzymatic antioxidant defence, but are still very closely associated with the subtle regulation of cellular redox balance. Related to the cardiometabolic health, the effects of polyphenols on cellular processes that are involved in chronic inflammation, endothelial dysfunction, impaired insulin signalling, adipose tissue remodelling or mitochondrial dysfunction have been reviewed in detail $^{(79-82)}$. Similar to what has been demonstrated for Keap1, there is growing body of scientific evidence that molecular mechanisms of health promoting effects of polyphenols involve their binding to specific proteins, and subsequent effects on cell signalling. For instance, in silico docking analyses revealed that curcumin also has the ability to bind to some kinases, such as TGF- $\beta$-activated kinase 1 (mitogen-activated protein kinase kinase kinase 7), 3-phosphoinositide-dependent protein kinase 1, serine/threonine kinase (AKT) 1 and AKT2, which are involved in regulation of the redox-sensitive pro-inflammatory transcription factor NF- $\kappa \mathrm{B}$. Indeed, NF- $\mathrm{BB}$ is established as a key mediator of inflammation, which is a common underlying mechanism of cardiometabolic diseases. Accordingly, a preexposure of human umbilical vein endothelial cells to curcumin $(1 \mu \mathrm{M})$ prior to $\mathrm{TNF} \alpha$ stimulation attenuates the activation of NF- $\kappa$ B. In parallel, curcumin decreases monocyte adhesion and transendothelial migration and modulates the expression of genes involved in antioxidant defence, metabolism, cell signalling, focal adhesion, intercellular junction and cytoskeleton organisation $^{(83)}$, which is altogether pertinent to an improved endothelial function. Similarly, it has been demonstrated that pre-exposure of human umbilical vein endothelial cells to physiologically relevant concentrations of plasma anthocyanins' metabolites (including their gut microbiota metabolites) also decreases the TNF $\alpha$-induced monocyte cell adhesion. Gene expression analysis demonstrated modulation of genes that are involved in cell adhesion, leucocyte transendothelial migration, regulation of actin cytoskeleton or NF- $\mathrm{KB}$ signalling. Subsequent bioinformatic analysis and in silico docking demonstrated that anthocyanins' metabolites can bind to several cell signalling proteins, including dual specificity mitogenactivated protein kinase kinase 2 and NF- $\kappa \mathrm{B}$ inhibitor kinase alpha, and potentially modulate their activity. The results of in silico docking were confirmed with Western blot analysis, demonstrating a decreased phosphorylation of mitogenactivated protein kinase and p65 unit of NF- $\mathrm{kB}$, pertinent to the modulation of upstream kinases, and the degreased leucocyte transendothelial migration and inflammation ${ }^{(84)}$. In addition, it has been demonstrated that flavanol colonic metabolites activate endothelial nitric oxide synthase in human endothelial cells via AKT and AMP-activated protein kinase signalling and increase intracellular nitric oxide, which plays a pivotal role in regulation of endothelial function ${ }^{(85)}$. An integrated systems' biology approach has been taken recently to elucidate the multi-target and multi-layer cellular effects of circulating epicatechin metabolites on endothelial cells, demonstrating modulation in gene and microRNA expressions and DNA methylation. In silico docking demonstrated favourable binding of epicatechin metabolites to p38 mitogen-activated protein kinase, which regulates several transcription factors such as NF- $\mathrm{BB}$, CREB1, c-MYC, c-JUN, STAT3 or SP1 ${ }^{(86)}$.

Molecular mechanisms of the effects of polyphenols on cardiometabolic health have also been studied in other relevant cell models, such as immune cells, adipocytes, hepatocytes, smooth muscle cells and $\beta$-cells. Aiming to systematise the present scientific evidence, we recently extracted and analysed the nutrigenomic data related to the effects of flavanols or their circulating metabolites in these cell models (paper under submission). Thereby, it was noticed that in large number of studies, the cells were exposed to plant extracts or high concentrations of pure compounds, which are not physiologically relevant ${ }^{(73)}$. This knowledge gap needs to be addressed in future, in order to better understand the molecular mechanisms of health promoting effects of polyphenols.

Regarding the contribution of nutritional polyphenols to the cellular antioxidant defence, an important consideration is that they are present in the cells at low concentrations. Therefore, they cannot scavenge a significant amount of free radicals. Instead, they modulate transcription of the genes involved in antioxidant defence and detoxification. On the other hand, if present at high concentrations, polyphenols may induce toxicity. These considerations are integrated in the concept of hormesis ${ }^{(87)}$, which also applies to nutritional polyphenols ${ }^{(88)}$. Potential toxicity of high doses of polyphenols should be always kept in mind, as over-the-counter supplements (such as resveratrol, curcumin, quercetin or various plant extracts) are widely available. The lesson learned from the antioxidant supplementation studies that 'natural' does not always mean 'safe in high doses' should be well remembered. As new strategies to increase the bioavailability of polyphenols are developing rapidly ${ }^{(89)}$, safety and toxicity should be carefully investigated. The topic of safety and toxicity ${ }^{(90)}$ becomes even more important when we take into account the inter-individual variability in response, which will be discussed in the following sections. 


\section{Effects of polyphenols on cardiometabolic health and cardiometabolic risk factors}

\section{Human intervention studies}

Following the pioneering study of Hertog et al. ${ }^{(59)}$, which demonstrated an inverse association between flavonoid intake and mortality from CHD, numerous human studies have been conducted to explore the impact of consumption of polyphenols on cardiometabolic health. In this type of studies, specific biomarkers should be clearly identified and precisely measured. It is above the scope of this review to analyse in detail this large body of scientific evidence. Therefore, in this paper, we present only several relevant human studies (Table 1). 'Cardiometabolic' is a relatively new term that encompasses a wide spectrum of cardiovascular and metabolic diseases, including CHD, non-alcoholic fatty liver disease, the metabolic syndrome and type 2 diabetes. These diseases are the leading cause of morbidity and mortality worldwide. They all share common risk factors such as overweight/obesity, inflammation, increased blood pressure and dyslipidaemia ${ }^{(91)}$. Knowing that diet and lifestyle largely determine the risk for cardiometabolic diseases, one of the main goals in promoting the cardiometabolic health is the development of preventive health programmes, such as adoption of healthy dietary habits by the majority of population. Moreover, personalised nutrition could be an effective strategy for prevention and treatment of cardiometabolic diseases in future, either at larger population groups or at individual level.

Cardioprotective effects of polyphenols have been extensively studied. In an acute study ${ }^{(92)}$, it has been demonstrated that flavonoid-rich apple improves plasma nitric oxide status and endothelial function (measured as flow-mediated dilatation) and lowers systolic blood pressure. These outcomes are among the most important determinants of cardiovascular health. One of the most convincing evidence for cardioprotective effects of dietary flavonoids has also been related to their beneficial effects on endothelial function, as demonstrated in a meta-analysis conducted in $2008^{(105)}$. In that sense, the most convincing effect was observed for cocoa flavonoids. Polyphenols have been pointed out as a promising nutritional approach for prevention and treatment of hypertension. Indeed, a large cross-sectional study demonstrated a negative correlation between total polyphenol intake and blood pressure, both systolic and diastolic ${ }^{(93)}$. Similarly, regular consumption of cocoa product rich in cocoa fibre significantly decreased the systolic and diastolic blood pressure in moderately hypercholesterolaemic humans ${ }^{(94)}$. Another important determinant of cardiovascular (and more broadly, cardiometabolic) health is the level of systemic inflammation of low intensity, which is clinically assessed using plasma C-reactive protein as a biomarker, preferably with a method of high sensitivity (hs-CRP). In that sense, a recent meta-analysis of RCT demonstrated that resveratrol significantly decreases serum CRP levels ${ }^{(106)}$, thus potentially decreasing the cardiovascular risk.

Cardioprotective effects of nutritional polyphenols are also mediated through their beneficial influence on circulating lipoproteins. As such, nutritional polyphenols exhibit a potential to decrease the atherogenic risk. The effect of epigallocatechin gallate on LDL-cholesterol was summarised in a recent systematic review, demonstrating a significant reduction of plasma LDL-cholesterol in healthy individuals with epigallocatechin gallate intake between 107 and $856 \mathrm{mg} / \mathrm{d}^{(107)}$. It has also been demonstrated that regular consumption of cocoa increases HDL-cholesterol and decreases oxidised LDL in elderly at high risk for $\mathrm{CVD}^{(95)}$. Similarly, regular consumption of flavanol-rich cocoa products has been found to increase HDL-cholesterol in both normocholesterolaemic and moderately hypercholesterolaemic free-living subjects ${ }^{(96,97)}$. In addition, increase in HDLcholesterol and decrease in plasma-oxidised LDL are linear with the phenolic content of olive oil ${ }^{(98)}$. Dyslipidaemia and oxidative stress, which are well-defined risk factors for atherosclerosis, are also decreased by diet containing products naturally rich in polyphenols $^{(99)}$.

Effects of dietary polyphenols on the metabolic component of the cardiometabolic risk factors have also been explored by many researchers. Indeed, the beneficial effects of dietary polyphenols on insulin sensitivity, glucose tolerance and insulin secretion may potentially decrease the risk of type 2 diabetes. In that sense, it has been demonstrated that strawberry and cranberry polyphenols improve insulin sensitivity in overweight and obese non-diabetic, insulin-resistant persons ${ }^{(100)}$ and that polyphenols in general decrease blood glucose response during the oral glucose tolerance test in individuals at high cardiometabolic risk ${ }^{(101)}$. Consumption of flavanol-rich dark chocolate ${ }^{(102)}$ and supplementation with epicatechin ${ }^{(103)}$ improve insulin sensitivity in healthy subjects. Importantly, numerous epidemiological studies and RCT have demonstrated that consumption of green tea, red wine and cocoa lowers the risk of type 2 diabetes $^{(108)}$. In addition, higher consumption of anthocyanins and anthocyanin-rich fruit has also been associated with lower risk of type 2 diabetes ${ }^{(104)}$.

Accumulated scientific evidence about the effects of cocoa flavanols and polyphenols in olive on specific aspects of human health was analysed by European Food Safety Authority Panel on Dietetic Products, Nutrition and Allergies. Regarding the cocoa flavanols ${ }^{(109,110)}$, the Panel endorses the health claim that they 'help maintain endothelium-dependent vasodilatation which contributes to healthy blood flow ${ }^{(110)}$, and regarding the polyphenols in olive, the Panel endorses that 'consumption of olive oil polyphenols contributes to the protection of blood lipids from oxidative damage,(111). Notably, although many human intervention studies demonstrated beneficial effects of polyphenols on cardiometabolic health, and predominantly on cardiometabolic risk factors, there are also studies that reported lack of expected outcomes. For example, red wine polyphenols did not improve obesityassociated insulin resistance ${ }^{(112)}$, and regular consumption of polyphenol-rich apple failed to influence endothelial function in hypercholesterolaemic adults ${ }^{(113)}$. Considering these and other similar findings, it is essential to understand that many aspects should be taken into account in order to predict the effects of dietary polyphenols on cardiometabolic health. The chemical variety of polyphenols, as well as the variety of their nutritional sources and food matrices, largely influences the effects of polyphenols in humans. In addition, the interindividual variability in bioavailability (which is governed by absorption, distribution, metabolism and excretion), 


\begin{tabular}{|c|c|c|c|c|c|c|c|c|c|c|}
\hline & Ð & $\widehat{\omega}$ & & E & $\widehat{\mathbb{N}}$ & 畣 & 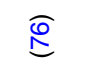 & & $E$ & న్ల్ర \\
\hline &  & 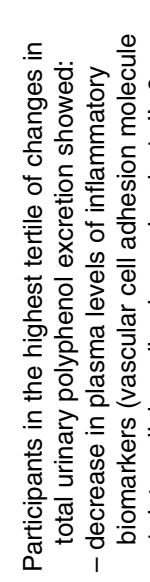 & 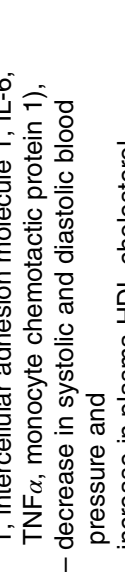 & 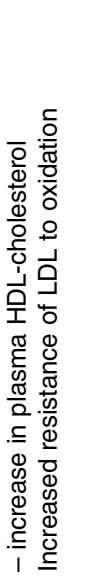 & 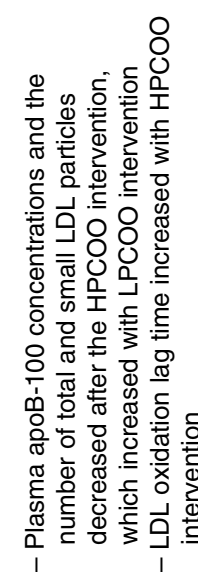 & 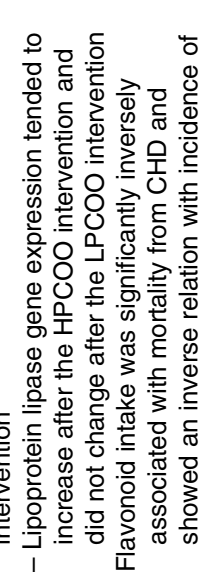 & 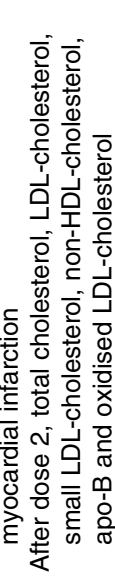 & 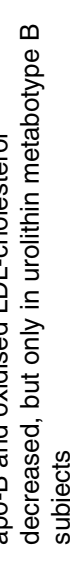 & 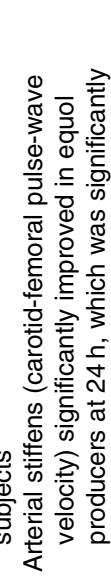 & 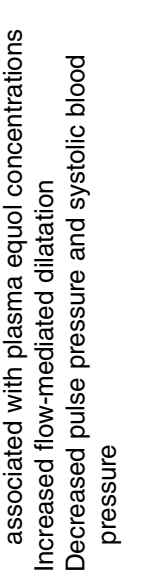 \\
\hline & 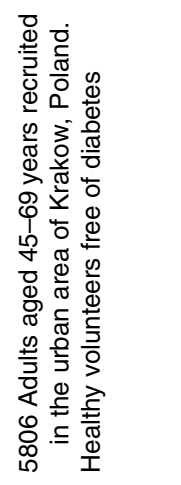 & 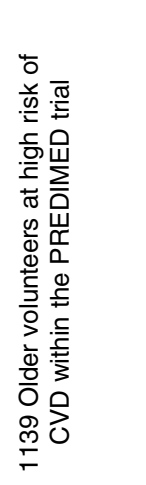 & & 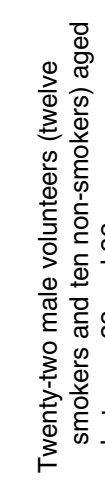 & 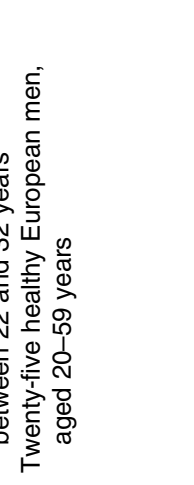 & 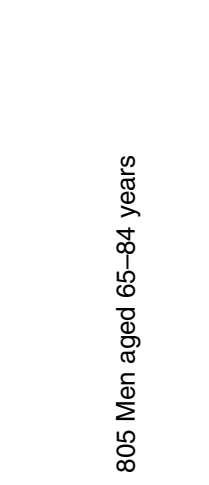 & 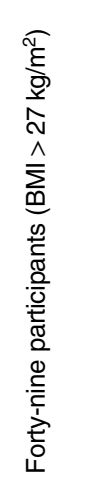 & & 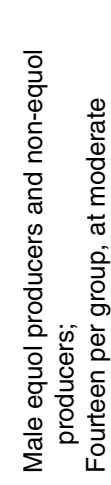 & 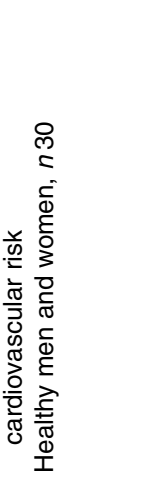 \\
\hline & 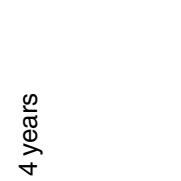 & 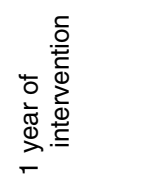 & & 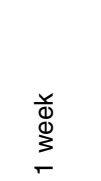 & 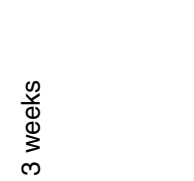 &  & 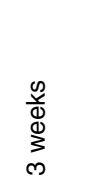 & & 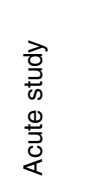 & 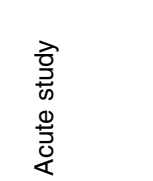 \\
\hline & 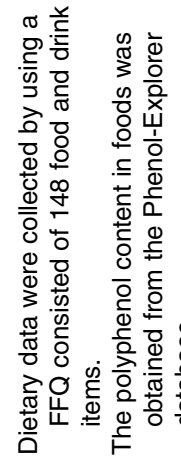 & 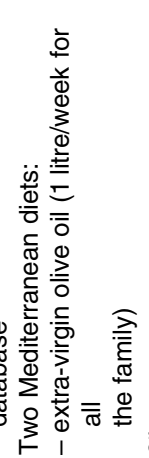 & 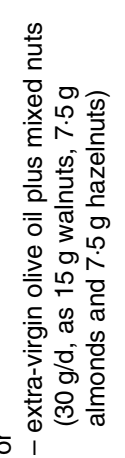 & 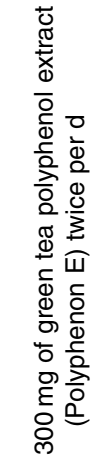 & 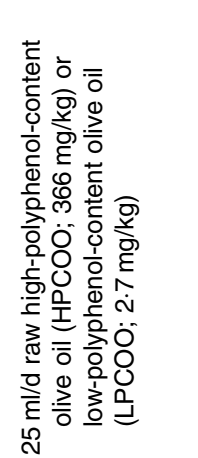 & 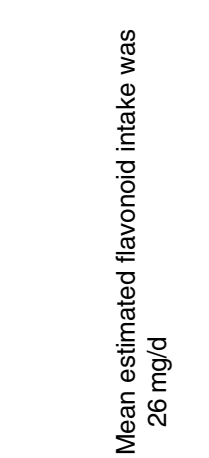 & 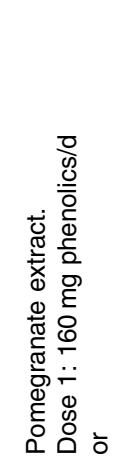 & 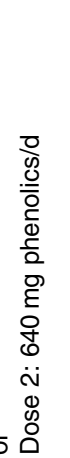 & 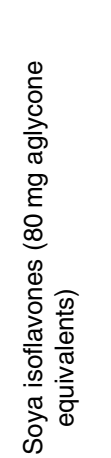 & 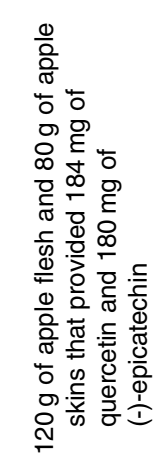 \\
\hline & 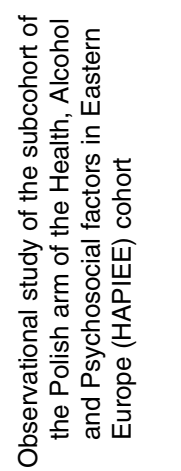 & 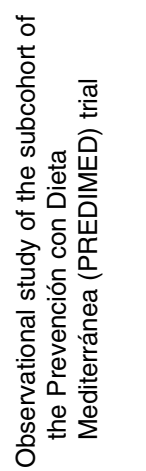 & & 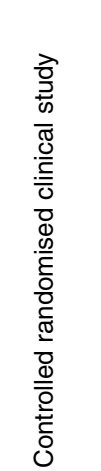 & 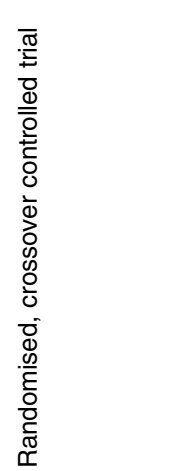 & 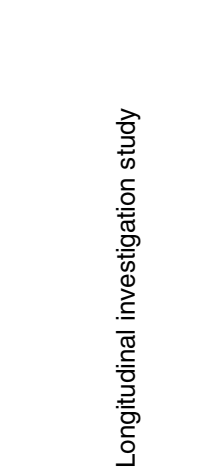 & 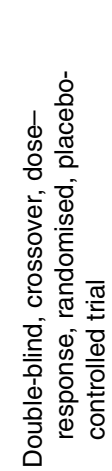 & & 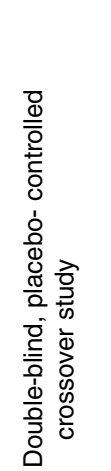 &  \\
\hline
\end{tabular}




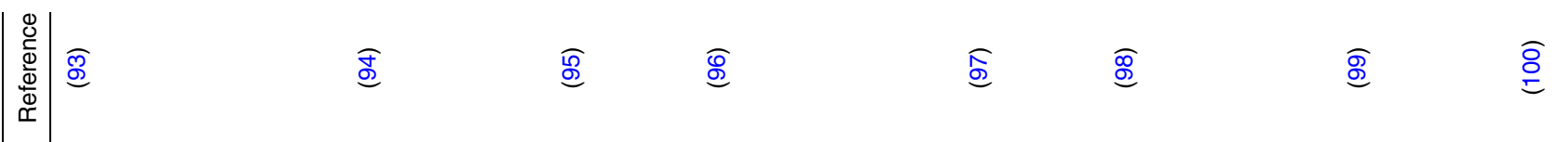


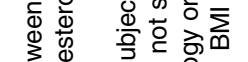

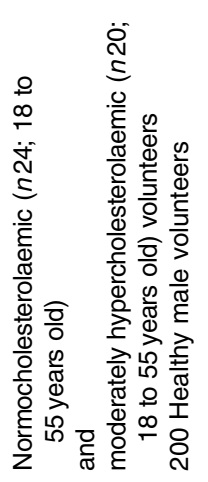


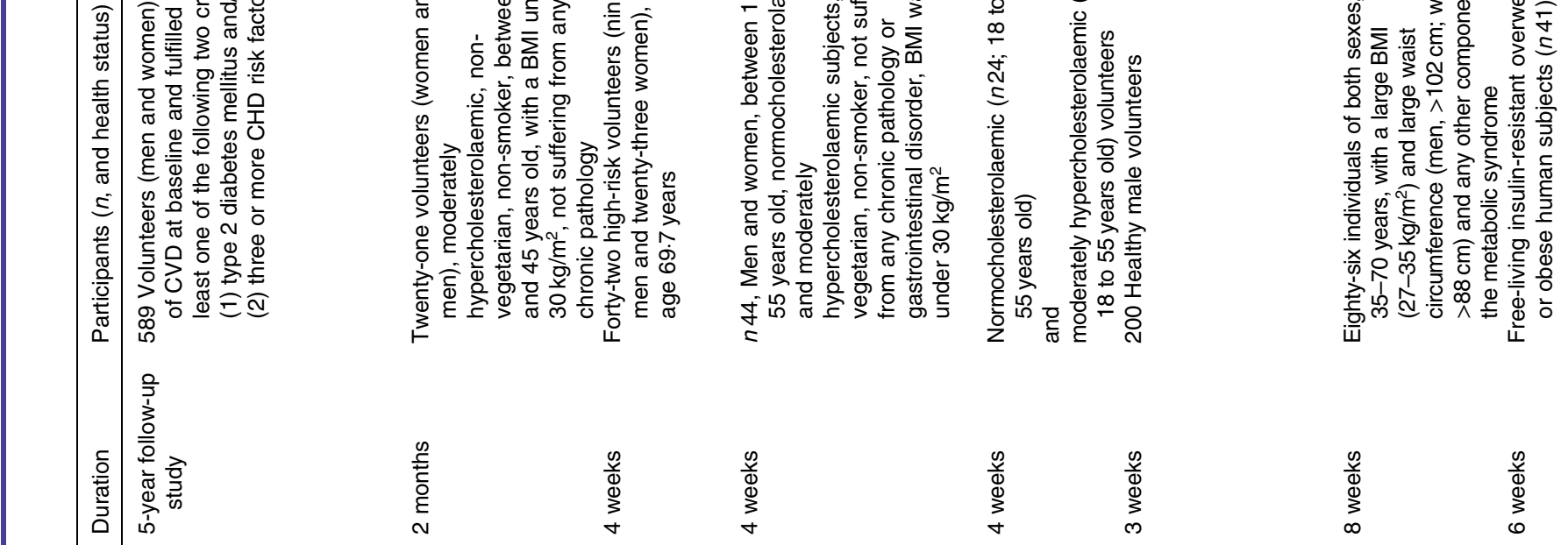

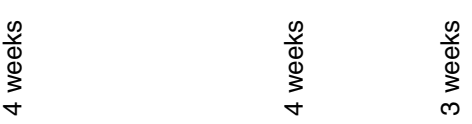

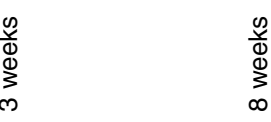

$\underset{\substack{n \\ \mathbb{D}}}{\stackrel{\infty}{\infty}}$
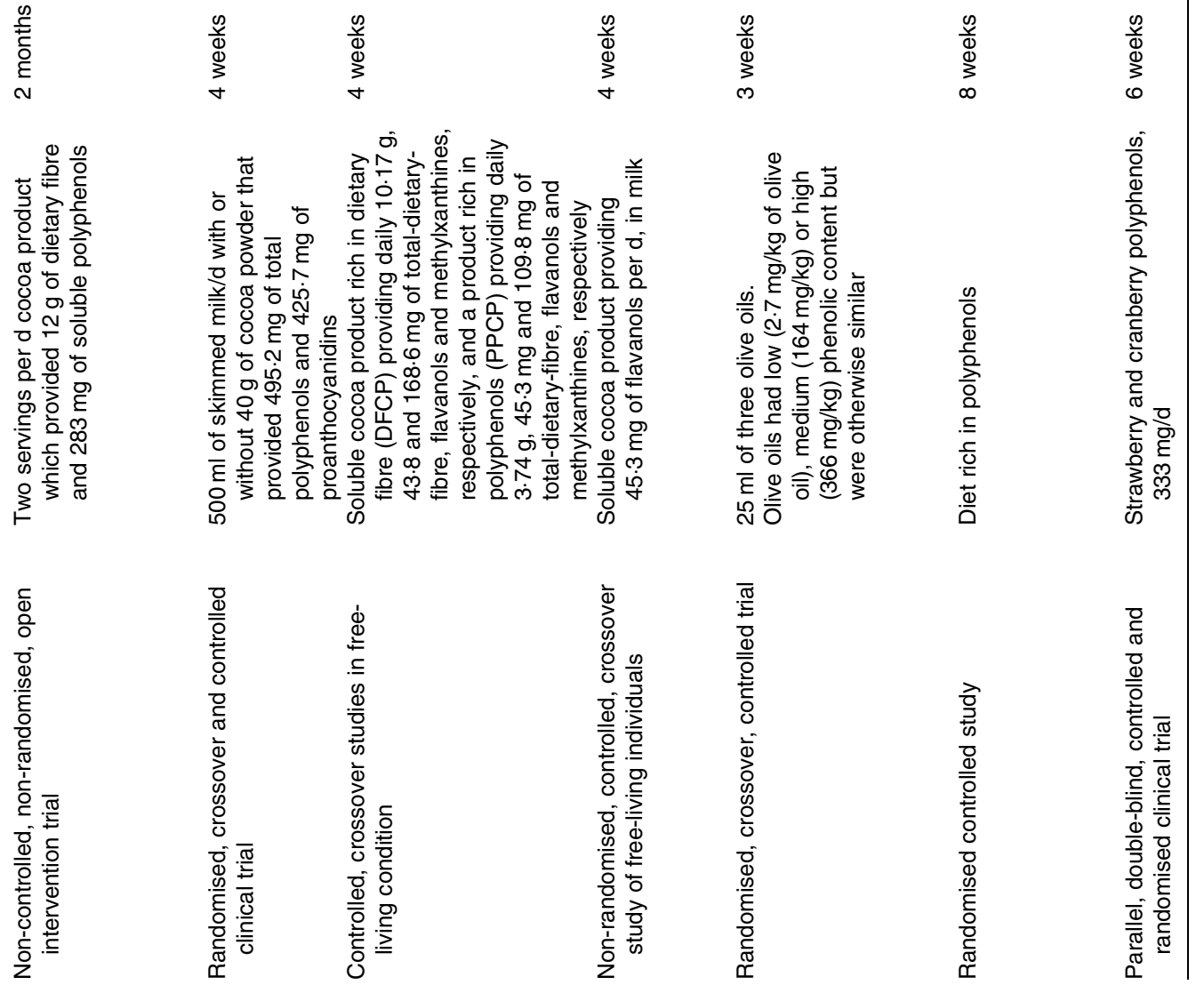
흘

$\simeq$

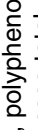

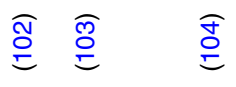



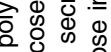

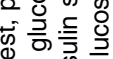

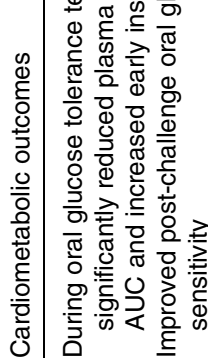

흘 흥

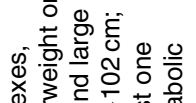

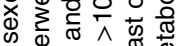

ᄃ วิธี



西

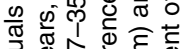

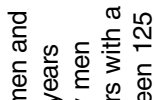
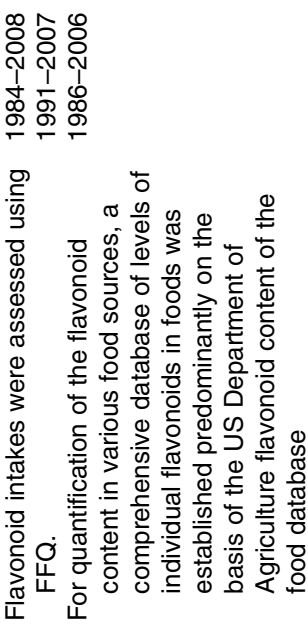

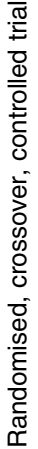

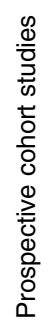

as well as the inter-individual variability in bioactivity of (the bioavailable) dietary polyphenols, undoubtedly play a pivotal role ${ }^{(114)}$. Besides, the inconsistency of results from human studies might be influenced, at least in part, by the heterogeneity of study populations, study designs and intervention periods.

\section{Inter-individual variability in response to consumption of polyphenols}

Ideally, dietary recommendations for intake of polyphenols (and plant food bioactives in general) should be determined by their (a) bioavailability and (b) bioactivity, taking into account the inter-individual variability. In the first place, the inter-individual variability of absorption, distribution, metabolism and excretion affects the bioavailability of polyphenols. Further, the bioactivity of the bioavailable polyphenols (and/ or their metabolites) is most likely additionally affected by the inter-individual variability of their cellular molecular metabolism in the target tissues, as well as the inter-individual variability of the cellular processes they modulate. All these factors together affect the inter-individual variability in response to consumption of polyphenols. Furthermore, age, sex, health status, genetics and epigenetic factors have been suggested as the main determinants of the inter-individual variability of response ${ }^{(115)}$. However, the specific contribution of each of these factors is not completely understood. Indeed, we are at the very early stage of the research in this field. The information we currently have about the inter-individual variability was mainly generated during the post hoc analyses, not from the studies that had initially been designed to study this subject. This issue should be well addressed in future.

To obtain more information about the inter-individual variability in cardiometabolic response of consumption of nutritional polyphenols, four meta-analyses were conducted recently, using the available data from the literature. The meta-analysis of the cardiometabolic health effects of flavonols confirmed that they improve plasma lipid status and decrease systolic and diastolic blood pressure and plasma glucose. Deeper study of the available data, aiming to address the inter-individual variability, demonstrated that these effects are more pronounced in participants with diagnosed disease or dyslipidaemia $^{(116)}$. The meta-analysis on flavanols also confirmed that they beneficially modulate several cardiometabolic risk factors, such as plasma lipids, BMI and waist circumference. However, higher efficacy was demonstrated in the participants with BMI $\geq 25 \mathrm{~kg} / \mathrm{m}^{2}$ and in non-medicated individuals, revealing the inter-individual variability ${ }^{(117)}$. Similarly, the beneficial effects of ellagitannins and anthocyanins on selected cardiometabolic risk factors were found to be more prominent in overweight and obese subjects ${ }^{(118)}$, and the effects of hydroxycinnamic acids may be greater in individuals at higher cardiometabolic risk ${ }^{(119)}$.

These meta-analyses are of high importance because they introduce and emphasise the significance of the inter-individual variability in response to consumption of dietary polyphenols to the scientific community. However, their main limitations were the following: (a) unavailability of individual data, pre- and post-intervention and (b) low number of studies. Importantly, even though facing these limitations, the authors were able to demonstrate significant inter-individual variability of several 

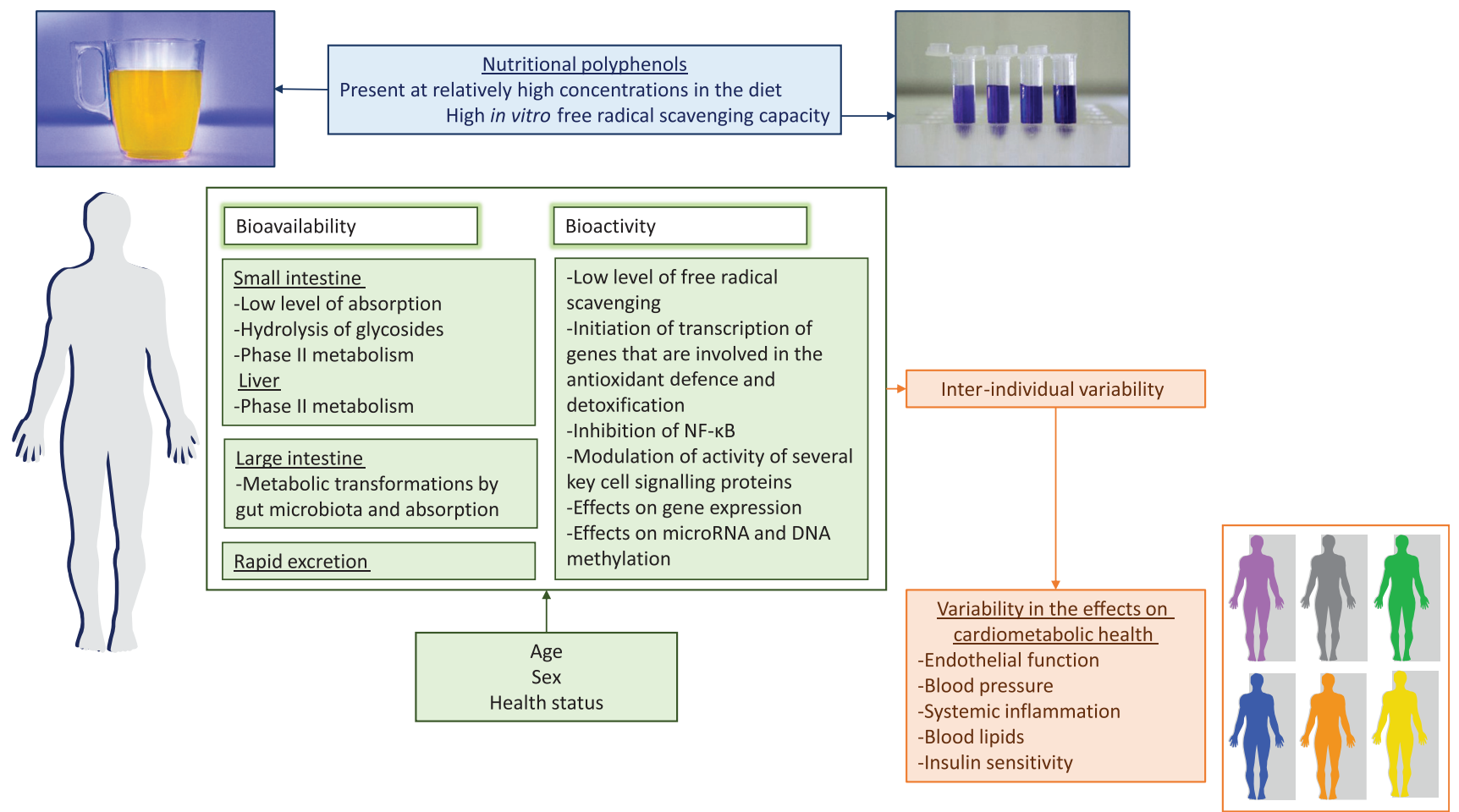

Fig. 1. Polyphenols in the human diet - in vitro and in vivo.

crucial cardiometabolic risk factors, which opens perspectives for further research in the field. However, in order to be able to conduct more robust meta-analyses in future, availability of individual data should be encouraged, of course in compliance with ethical standards. Understanding the factors that determine the inter-individual variability in response to consumption of polyphenols will lead us towards tailoring of personalised dietary recommendations, at both population group and individual level.

\section{Conclusion and perspective}

In this review, we aim to address the key aspects of the impact of polyphenols on human cardiometabolic health, historically and in perspective, as summarised in Fig. 1. Historically, there was a tendency to attribute the positive effects of polyphenols on human cardiometabolic health exclusively to their free radical scavenging capacity. Nowadays, we understand that they express their antioxidant activity mainly through modulation of transcription of genes involved in antioxidant defence and detoxification. Apart from their antioxidant activity, polyphenols also contribute to the cardiometabolic health by modulating a plethora of cellular processes that are not directly associated with the antioxidant enzymes, which are subject to extensive research. In the cells, dietary polyphenols and/or their metabolites are present at low concentrations. Administered at very high concentrations, polyphenols may cause toxic effects in humans. Informed about the beneficial effects of polyphenols on human cardiometabolic health, the temptation among the general population for taking supplements of pure compounds or plant extracts, which are readily available as over-thecounter pharmaceutical forms, is realistic. However, the vigilance for their potential toxicity, if taken at high doses, should remain high.

Growing scientific evidence demonstrates the beneficial effects of dietary polyphenols on human cardiometabolic health. Numerous RCT report improvement of the key biomarkers of cardiometabolic health such as blood pressure, flow-mediated dilatation, blood lipids, blood glucose and insulin sensitivity. The inconsistency of the results of some studies triggered interest towards post hoc analysis of individual data, which led to identification of the inter-individual variability in the response. Resolving the puzzle of the inter-individual variability will lead us towards personalised dietary recommendations, with ultimate goal to decrease the risk for cardiometabolic diseases and preserve the health for better ageing. For this purpose, future studies shall be specifically designed to address the various aspects of the inter-individual variability in response to consumption of polyphenols and plant food bioactives in general.

\section{Acknowledgements}

The authors are members of COST Action FA1403 - POSITIVe 'Interindividual variation in response to consumption of plant food bioactives and determinants involved' supported by COST (European Cooperation in Science and Technology, http://www.cost.eu/).

The authors thank Kosta Zamanovski and Ljubica Janeska for original photographs and figure elements. 
This research received no specific grant from any funding agency, commercial or not-for-profit sectors.

T. R., V. M. and D. M. conceived the structure, wrote the manuscript and approved the final version.

There are no conflicts of interest.

\section{References}

1. Anderson AS (2007) Nutrition interventions in women in low-income groups in the UK. Proc Nutr Soc 66, 25-32.

2. Dammann KW \& Smith C (2009) Factors affecting low-income women's food choices and the perceived impact of dietary intake and socioeconomic status on their health and weight. J Nutr Educ Behav 41, 242-253.

3. Barker M, Lawrence W, Crozier S, et al. (2009) Educational attainment, perceived control and the quality of women's diets. Appetite 52, 631-636.

4. Grosso G, Stepaniak U, Micek A, et al. (2017) Dietary polyphenol intake and risk of type 2 diabetes in the Polish arm of the Health, Alcohol and Psychosocial factors in Eastern Europe (HAPIEE) study. Br J Nutr 118, 60-68.

5. Medina-Remon A, Casas R, Tressserra-Rimbau A, et al. (2017) Polyphenol intake from a Mediterranean diet decreases inflammatory biomarkers related to atherosclerosis: a sub study of the PREDIMED trial. Br J Clin Pharmacol 83 114-128.

6. Sawikr Y, Yarla NS, Peluso I, et al. (2017) Neuroinflammation in Alzheimer's disease: the preventive and therapeutic potential of polyphenolic nutraceuticals. Adv Protein Chem Struct Biol 108, 33-57.

7. Costa C, Tsatsakis A, Mamoulakis C, et al. (2017) Current evidence on the effect of dietary polyphenols intake on chronic diseases. Food Chem Toxicol 110, 286-299.

8. Salah N, Miller NJ, Paganga G, et al. (1995) Polyphenolic flavanols as scavengers of aqueous phase radicals and as chain-breaking antioxidants. Arch Biochem Biophys 322 , 339-346.

9. Visioli F, Bellomo G \& Galli C (1998) Free radical-scavenging properties of olive oil polyphenols. Biochem Biophys Res Commun 247, 60-64.

10. Vinson JA (1998) Flavonoids in foods as in vitro and in vivo antioxidants. Adv Exp Med Biol 439, 151-164.

11. Miura Y, Chiba T, Miura S, et al. (2000) Green tea polyphenols (flavan 3-ols) prevent oxidative modification of low density lipoproteins: an ex vivo study in humans. J Nutr Biochem 11, 216-222.

12. Hernaez A, Remaley AT, Farras M, et al. (2015) Olive oil polyphenols decrease LDL concentrations and LDL atherogenicity in men in a randomized controlled trial. $J$ Nutr $\mathbf{1 4 5}$, 1692-1697.

13. Kehrer JP \& Klotz LO (2015) Free radicals and related reactive species as mediators of tissue injury and disease: implications for health. Crit Rev Toxicol 45, 765-798.

14. Harman D (1956) Aging: a theory based on free radical and radiation chemistry. J Gerontol 11, 298-300.

15. Paniker NV, Srivastava SK \& Beutler E (1970) Glutathione metabolism of the red cells. Effect of glutathione reductase deficiency on the stimulation of hexose monophosphate shunt under oxidative stress. Biochim Biophys Acta 215, 456-460.

16. Sies H (1985) Oxidative stress: introductory remarks. In Oxidative Stress, pp. 1-8 [H Sies, editor]. London: Academic Press, Elsevier Ltd.

17. Sies H \& Cadenas E (1985) Oxidative stress: damage to intact cells and organs. Philos Trans R Soc Lond B Biol Sci 311, 617-631.
18. Jones DP (2006) Redefining oxidative stress. Antioxid Redox Signal 8, 1865-1879.

19. Jones DP (2008) Radical-free biology of oxidative stress. Am J Physiol Cell Physiol 295, C849-C868.

20. Sies H (2019) Oxidative stress: eustress and distress in redox homeostasis. In Stress: Physiology, Biochemistry, and Pathology, pp. 153-163 [G Fink, editor]. London: Academic Press, Elsevier Ltd.

21. Birben E, Sahiner UM, Sackesen C, et al. (2012) Oxidative stress and antioxidant defense. World Allergy Organ J 5, 9-19.

22. Zimniak P (2011) Relationship of electrophilic stress to aging. Free Radic Biol Med 51, 1087-1105.

23. Cipak Gasparovic A, Zarkovic N, Zarkovic K, et al. (2017) Biomarkers of oxidative and nitro-oxidative stress: conventional and novel approaches. BrJ Pharmacol 174, 1771-1783.

24. Ruskovska T \& Bernlohr DA (2013) Oxidative stress and protein carbonylation in adipose tissue - implications for insulin resistance and diabetes mellitus. J Proteomics 92, 323-334.

25. Gueraud F, Atalay M, Bresgen N, et al. (2010) Chemistry and biochemistry of lipid peroxidation products. Free Radic Res 44, 1098-1124.

26. Tsuchihashi H, Kigoshi M, Iwatsuki M, et al. (1995) Action of beta-carotene as an antioxidant against lipid peroxidation. Arch Biochem Biophys 323, 137-147.

27. Spiteller G (2002) Are changes of the cell membrane structure causally involved in the aging process? Ann N Y Acad Sci 959 , $30-44$.

28. Jiang Q, Christen S, Shigenaga MK, et al. (2001) Gammatocopherol, the major form of vitamin $\mathrm{E}$ in the US diet, deserves more attention. Am J Clin Nutr 74, 714-722.

29. Kurutas EB (2016) The importance of antioxidants which play the role in cellular response against oxidative/nitrosative stress: current state. Nutr J 15, 71.

30. Niki E (2014) Role of vitamin E as a lipid-soluble peroxyl radical scavenger: in vitro and in vivo evidence. Free Radic Biol Med 66, 3-12.

31. Bjelakovic G, Nikolova D, Gluud LL, et al. (2008) Antioxidant supplements for prevention of mortality in healthy participants and patients with various diseases. Cochrane Database Syst Rev, issue 2, CD007176.

32. Bjelakovic G, Nikolova D, Gluud LL, et al. (2012) Antioxidant supplements for prevention of mortality in healthy participants and patients with various diseases. Cochrane Database Syst Rev, issue 3, CD007176.

33. Ghezzi P, Jaquet V, Marcucci F, et al. (2017) The oxidative stress theory of disease: levels of evidence and epistemological aspects. Br J Pharmacol 174, 1784-1796.

34. Milkovic L, Gasparovic AC, Zarkovic N (2015) Overview on major lipid peroxidation bioactive factor 4-hydroxynonenal as pluripotent growth-regulating factor. Free Radic Res $\mathbf{4 9}$, 850-860.

35. Chen ZH, Saito Y, Yoshida Y, et al. (2005) 4-Hydroxynonenal induces adaptive response and enhances PC12 cell tolerance primarily through induction of thioredoxin reductase 1 via activation of Nrf2. J Biol Chem 280, 41921-41927.

36. Azzi A (2018) Many tocopherols, one vitamin E. Mol Aspects Med 61, 92-103.

37. von Lintig J (2012) Provitamin A metabolism and functions in mammalian biology. Am J Clin Nutr 96, 1234S-1244S.

38. Vrolijk MF, Opperhuizen A, Jansen EH, et al. (2015) The shifting perception on antioxidants: the case of vitamin $\mathrm{E}$ and $\beta$-carotene. Redox Biol 4, 272-278.

39. Alpha-Tocopherol, Beta Carotene Cancer Prevention Study Group (1994) The effect of vitamin E and beta carotene on the incidence of lung cancer and other cancers in male smokers. N Engl J Med 330, 1029-1035. 
40. Niki E (2014) Antioxidants: basic principles, emerging concepts, and problems. Biomed J 37, 106-111.

41. Sanyal AJ, Chalasani N, Kowdley KV, et al. (2010) Pioglitazone, vitamin E, or placebo for nonalcoholic steatohepatitis. $N$ Engl J Med 362, 1675-1685.

42. Vardi M, Levy NS \& Levy AP (2013) Vitamin E in the prevention of cardiovascular disease: the importance of proper patient selection. J Lipid Res 54, 2307-2314.

43. Ruskovska T, Pop-Kostova A, Jansen EHJM, et al. (2017) Vitamin E supplementation in chronically hemodialyzed patients - influence on blood hemoglobin and plasma (anti) oxidant status. Int J Vitam Nutr Res 87, 139-148.

44. Ahmad KA, Yuan Yuan D, Nawaz W, et al. (2017) Antioxidant therapy for management of oxidative stress induced hypertension. Free Radic Res 51, 428-438.

45. Abner EL, Schmitt FA, Mendiondo MS, et al. (2011) Vitamin E and all-cause mortality: a meta-analysis. Curr Aging Sci $\mathbf{4}$, $158-170$

46. Curtis AJ, Bullen M, Piccenna L, et al. (2014) Vitamin E supplementation and mortality in healthy people: a metaanalysis of randomised controlled trials. Cardiovasc Drugs Ther 28, 563-573.

47. Jansen E \& Ruskovska T (2015) Serum biomarkers of (anti)oxidant status for epidemiological studies. Int J Mol Sci. 16 , 27378-27390.

48. Frijhoff J, Winyard PG, Zarkovic N, et al. (2015) Clinical relevance of biomarkers of oxidative stress. Antioxid Redox Signal 23, 1144-1170.

49. Kurin E, Mucaji P \& Nagy M (2012) In vitro antioxidant activities of three red wine polyphenols and their mixtures: an interaction study. Molecules 17, 14336-14348.

50. Erben-Russ M, Bors W \& Saran M (1987) Reactions of linoleic acid peroxyl radicals with phenolic antioxidants: a pulse radiolysis study. Int J Radiat Biol Relat Stud Phys Chem Med 52, 393-412.

51. Siti HN, Kamisah Y, Kamsiah J (2015) The role of oxidative stress, antioxidants and vascular inflammation in cardiovascular disease (a review). Vascul Pharmacol 71, 40-56.

52. Gonzalez S, Fernandez M, Cuervo A, et al. (2014) Dietary intake of polyphenols and major food sources in an institutionalised elderly population. J Hum Nutr Diet 27, 176-183.

53. Miranda AM, Steluti J, Fisberg RM, et al. (2016) Dietary intake and food contributors of polyphenols in adults and elderly adults of Sao Paulo: a population-based study. Br J Nutr 115, 1061-1070.

54. Grosso G, Stepaniak U, Topor-Mądry R, et al. (2014) Estimated dietary intake and major food sources of polyphenols in the Polish arm of the HAPIEE study. Nutrition 30, 1398-1403.

55. Scalbert A \& Williamson G (2000) Dietary intake and bioavailability of polyphenols. J Nutr 130, 2073S-2085S.

56. Pandey KB \& Rizvi SI (2009) Plant polyphenols as dietary antioxidants in human health and disease. Oxid Med Cell Longev 2, 270-278.

57. Neveu V, Perez-Jimenez J, Vos F, et al. (2010) Phenolexplorer: an online comprehensive database on polyphenol contents in foods. Database (Oxford) 2010, bap024. http:// www.phenol-explorer.eu (accessed March 2019).

58. Bhagwat S, Haytowitz DB, \& Holden JM (ret.) (2014) USDA database for the flavonoid content of selected foods. Release 3.1. U.S. Department of Agriculture, Agricultural Research Service. http://www.ars.usda.gov/nutrientdata/flav (accessed March 2019).

59. Hertog MG, Feskens EJ, Hollman PC, et al. (1993) Dietary antioxidant flavonoids and risk of coronary heart disease: the Zutphen Elderly Study. Lancet 342, 1007-1011.
60. U.S. Department of Agriculture, Agricultural Research Service (2010) USDA Database for the Oxygen Radical Absorbance Capacity (ORAC) of Selected Foods. Release 2.

61. Prior RL, Hoang H, Gu L, et al. (2003). Assays for hydrophilic and lipophilic antioxidant capacity (oxygen radical absorbance capacity (ORAC(FL)) ) of plasma and other biological and food samples. J Agric Food Chem 51, 3273-3279.

62. Gillespie KM, Chae JM \& Ainsworth EA (2007) Rapid measurement of total antioxidant capacity in plants. Nat Protoc 2, 867-870.

63. Litescu SC, Eremia S \& Radu GL (2010) Methods for the determination of antioxidant capacity in food and raw materials. Adv Exp Med Biol 698, 241-249.

64. Cunningham E (2013) What has happened to the ORAC database? J Acad Nutr Diet 113, 740.

65. Manach C, Williamson G, Morand C, et al. (2005) Bioavailability and bioefficacy of polyphenols in humans. I. Review of 97 bioavailability studies. Am J Clin Nutr 81, 230S-242S.

66. Halliwell B, Zhao K \& Whiteman M (2000) The gastrointestinal tract: a major site of antioxidant action? Free Radic Res $\mathbf{3 3}$, 819-830.

67. Del Rio D, Rodriguez-Mateos A, Spencer JP, et al. (2013) Dietary (poly)phenolics in human health: structures, bioavailability, and evidence of protective effects against chronic diseases. Antioxid Redox Signal 18, 1818-1892.

68. Lotito SB, Zhang WJ, Yang CS, et al. (2011) Metabolic conversion of dietary flavonoids alters their antiinflammatory and antioxidant properties. Free Radic Biol Med 51, 454-463.

69. Loke WM, Proudfoot JM, Stewart S, et al. (2008) Metabolic transformation has a profound effect on anti-inflammatory activity of flavonoids such as quercetin: lack of association between antioxidant and lipoxygenase inhibitory activity. Biochem Pharmacol 75, 1045-1053.

70. Williamson G \& Clifford MN (2017) Role of the small intestine, colon and microbiota in determining the metabolic fate of polyphenols. Biochem Pharmacol 139, 24-39.

71. Mullen W, Edwards CA \& Crozier A (2006) Absorption, excretion and metabolite profiling of methyl-, glucuronyl-, glucosyl- and sulpho-conjugates of quercetin in human plasma and urine after ingestion of onions. Br J Nutr $\mathbf{9 6}$, 107-116.

72. Inoue-Choi M, Yuan JM, Yang CS, et al. (2010) Genetic association between the COMT genotype and urinary levels of tea polyphenols and their metabolites among daily green tea drinkers. Int J Mol Epidemiol Genet 1, 114-123.

73. Gibney ER, Milenkovic D, Combet E, et al. (2019) Factors influencing the cardiometabolic response to (poly)phenols and phytosterols: a review of the COST Action POSITIVe activities. Eur J Nutr 58, Suppl. 2, 37-47.

74. Kawabata K, Yoshioka Y \& Terao J (2019) Role of intestinal microbiota in the bioavailability and physiological functions of dietary polyphenols. Molecules 24, E370.

75. Fernandez-Millan E, Ramos S, Alvarez C, et al. (2014) Microbial phenolic metabolites improve glucose-stimulated insulin secretion and protect pancreatic beta cells against tert-butyl hydroperoxide-induced toxicity via ERKs and PKC pathways. Food Chem Toxicol 66, 245-253.

76. Gonzalez-Sarrias A, Garcia-Villalba R, Romo-Vaquero M, et al. (2017) Clustering according to urolithin metabotype explains the interindividual variability in the improvement of cardiovascular risk biomarkers in overweight-obese individuals consuming pomegranate: a randomized clinical trial. $\mathrm{Mol} \mathrm{Nutr}$ Food Res 61, 1600830. 
77. Hazim S, Curtis PJ, Schar MY, et al. (2016) Acute benefits of the microbial-derived isoflavone metabolite equol on arterial stiffness in men prospectively recruited according to equol producer phenotype: a double-blind randomized controlled trial. Am J Clin Nutr 103, 694-702.

78. Forman HJ, Davies KJ \& Ursini F (2014) How do nutritional antioxidants really work: nucleophilic tone and para-hormesis versus free radical scavenging in vivo. Free Radic Biol Med 66, 24-35.

79. Krga I, Milenkovic D, Morand C, et al. (2016) An update on the role of nutrigenomic modulations in mediating the cardiovascular protective effect of fruit polyphenols. Food Funct 7, 3656-3676.

80. Kim HS, Quon MJ \& Kim JA (2014) New insights into the mechanisms of polyphenols beyond antioxidant properties; lessons from the green tea polyphenol, epigallocatechin 3-gallate. Redox Biol 2, 187-195.

81. Goszcz K, Duthie GG, Stewart D, et al. (2017) Bioactive polyphenols and cardiovascular disease: chemical antagonists, pharmacological agents or xenobiotics that drive an adaptive response? Br J Pharmacol 174, 1209-1225.

82. Kerimi A \& Williamson G (2016) At the interface of antioxidant signalling and cellular function: key polyphenol effects. $\mathrm{Mol}$ Nutr Food Res 60, 1770-1788.

83. Monfoulet LE, Mercier S, Bayle D, et al. (2017) Curcumin modulates endothelial permeability and monocyte transendothelial migration by affecting endothelial cell dynamics. Free Radic Biol Med 112, 109-120.

84. Krga I, Tamaian R, Mercier S, et al. (2018) Anthocyanins and their gut metabolites attenuate monocyte adhesion and transendothelial migration through nutrigenomic mechanisms regulating endothelial cell permeability. Free Radic Biol Med 124, 364-379.

85. Alvarez-Cilleros D, Ramos S, Goya L, et al. (2018) Colonic metabolites from flavanols stimulate nitric oxide production in human endothelial cells and protect against oxidative stress-induced toxicity and endothelial dysfunction. Food Chem Toxicol 115, 88-97.

86. Milenkovic D, Berghe WV, Morand C, et al. (2018) A systems biology network analysis of nutri(epi)genomic changes in endothelial cells exposed to epicatechin metabolites. Sci Rep 8, 15487.

87. Rattan S (2014) Aging, health, hormesis and future lines of investigation. Aging Sci 2, 1000e111.

88. Son TG, Camandola S \& Mattson MP (2008) Hormetic dietary phytochemicals. Neuromolecular Med 10, 236-246.

89. Lewandowska U, Szewczyk K, Hrabec E, et al. (2013) Overview of metabolism and bioavailability enhancement of polyphenols. J Agric Food Chem 61, 12183-12199.

90. Yates AA, Erdman JW Jr, Shao A, et al. (2017) Bioactive nutrients - time for tolerable upper intake levels to address safety. Regul Toxicol Pharmacol 84, 94-101.

91. Guo F, Moellering RD \& Garvey WT (2014) The progression of cardiometabolic disease: validation of a new cardiometabolic disease staging system applicable to obesity. Obesity (Silver Spring) 22, 110-118.

92. Bondonno CP, Yang X, Croft KD, et al. (2012) Flavonoid-rich apples and nitrate-rich spinach augment nitric oxide status and improve endothelial function in healthy men and women: a randomized controlled trial. Free Radic Biol Med 52, 95-102.

93. Medina-Remon A, Zamora-Ros R, Rotches-Ribalta M, et al. (2011) Total polyphenol excretion and blood pressure in subjects at high cardiovascular risk. Nutr Metab Cardiovasc Dis 21, 323-331.
94. Sarria B, Mateos R, Sierra-Cinos JL, et al. (2012) Hypotensive, hypoglycaemic and antioxidant effects of consuming a cocoa product in moderately hypercholesterolemic humans. Food Funct 3, 867-874.

95. Khan N, Monagas M, Andres-Lacueva C, et al. (2012) Regular consumption of cocoa powder with milk increases HDL cholesterol and reduces oxidized LDL levels in subjects at high-risk of cardiovascular disease. Nutr Metab Cardiovasc Dis 22, 1046-1053.

96. Sarria B, Martinez-Lopez S, Sierra-Cinos JL, et al. (2015) Effects of bioactive constituents in functional cocoa products on cardiovascular health in humans. Food Chem 174, 214-218.

97. Martinez-Lopez S, Sarria B, Sierra-Cinos JL, et al. (2014) Realistic intake of a flavanol-rich soluble cocoa product increases HDL-cholesterol without inducing anthropometric changes in healthy and moderately hypercholesterolemic subjects. Food Funct 5, 364-374.

98. Covas MI, Nyyssonen K, Poulsen HE, et al. (2006) The effect of polyphenols in olive oil on heart disease risk factors: a randomized trial. Ann Intern Med 145, 333-341.

99. Annuzzi G, Bozzetto L, Costabile G, et al. (2014) Diets naturally rich in polyphenols improve fasting and postprandial dyslipidemia and reduce oxidative stress: a randomized controlled trial. Am J Clin Nutr 99, 463-471.

100. Paquette M, Medina Larque AS, Weisnagel SJ, et al. (2017) Strawberry and cranberry polyphenols improve insulin sensitivity in insulin-resistant, non-diabetic adults: a parallel, double-blind, controlled and randomised clinical trial. $\mathrm{Br} J$ Nutr 117, 519-531.

101. Bozzetto L, Annuzzi G, Pacini G, et al. (2015) Polyphenol-rich diets improve glucose metabolism in people at high cardiometabolic risk: a controlled randomised intervention trial. Diabetologia 58, 1551-1560.

102. Grassi D, Lippi C, Necozione S, et al. (2005) Short-term administration of dark chocolate is followed by a significant increase in insulin sensitivity and a decrease in blood pressure in healthy persons. Am J Clin Nutr 81, 611-614.

103. Dower JI, Geleijnse JM, Gijsbers L, et al. (2015) Effects of the pure flavonoids epicatechin and quercetin on vascular function and cardiometabolic health: a randomized, doubleblind, placebo-controlled, crossover trial. Am J Clin Nutr 101, 914-921.

104. Wedick NM, Pan A, Cassidy A, et al. (2012) Dietary flavonoid intakes and risk of type 2 diabetes in US men and women. Am J Clin Nutr 95, 925-933.

105. Hooper L, Kroon PA, Rimm EB, et al. (2008) Flavonoids, flavonoid-rich foods, and cardiovascular risk: a meta-analysis of randomized controlled trials. Am J Clin Nutr 88, 38-50.

106. Haghighatdoost F \& Hariri M (2019) Can resveratrol supplement change inflammatory mediators? A systematic review and meta-analysis on randomized clinical trials. Eur J Clin Nutr 73, 345-355.

107. Momose Y, Maeda-Yamamoto M \& Nabetani H (2016) Systematic review of green tea epigallocatechin gallate in reducing low-density lipoprotein cholesterol levels of humans. Int J Food Sci Nutr 67, 606-613.

108. Martin MA, Goya L \& Ramos S (2017) Protective effects of tea, red wine and cocoa in diabetes. Evidences from human studies. Food Chem Toxicol 109, 302-314.

109. EFSA Panel on Dietetic Products, Nutrition and Allergies (NDA) (2010) Scientific Opinion on the substantiation of health claims related to cocoa flavanols and protection of lipids from oxidative damage (ID 652, 1372, 1506, 3143), and maintenance of normal blood pressure (ID 1507) pursuant to Article 13(1) of Regulation (EC) No 1924/2006. EFSA J 8, 1792 
110. EFSA Panel on Dietetic Products, Nutrition and Allergies (NDA) (2012) Scientific Opinion on the substantiation of a health claim related to cocoa flavanols and maintenance of normal endothelium-dependent vasodilation pursuant to Article 13(5) of Regulation (EC) No 1924/2006. EFSA J 10, 2809

111. EFSA Panel on Dietetic Products, Nutrition and Allergies (NDA) (2011) Scientific opinion on the substantiation of health claims related to polyphenols in olive and protection of LDL particles from oxidative damage (ID 1333, 1638, 1639, 1696, 2865), maintenance of normal blood HDLcholesterol concentrations (ID 1639), maintenance of normal blood pressure (ID 3781), 'anti-inflammatory properties' (ID 1882), 'contributes to the upper respiratory tract health' (ID 3468), 'can help to maintain a normal function of gastrointestinal tract' (3779), and 'contributes to body defences against external agents' (ID 3467) pursuant to Article 13(1) of Regulation (EC) No 1924/2006. EFSA J 9, 2033.

112. Woerdeman J, Del Rio D, Calani L, et al. (2018) Red wine polyphenols do not improve obesity-associated insulin resistance: a randomized controlled trial. Diabetes Obes Metab 20, 206-210.

113. Auclair S, Chironi G, Milenkovic D, et al. (2010) The regular consumption of a polyphenol-rich apple does not influence endothelial function: a randomised double-blind trial in hypercholesterolemic adults. Eur J Clin Nutr 64, $1158-1165$.
114. Manach C, Milenkovic D, Van de Wiele T, et al. (2017) Addressing the inter-individual variation in response to consumption of plant food bioactives: towards a better understanding of their role in healthy aging and cardiometabolic risk reduction. Mol Nutr Food Res 61, 1600557.

115. Milenkovic D, Morand C, Cassidy A, et al. (2017) Interindividual variability in biomarkers of cardiometabolic health after consumption of major plant-food bioactive compounds and the determinants involved. Adv Nutr 8, 558-570.

116. Menezes R, Rodriguez-Mateos A, Kaltsatou A, et al. (2017) Impact of flavonols on cardiometabolic biomarkers: a metaanalysis of randomized controlled human trials to explore the role of inter-individual variability. Nutrients 9, E117.

117. Gonzalez-Sarrias A, Combet E, Pinto P, et al. (2017) A systematic review and meta-analysis of the effects of flavanol-containing tea, cocoa and apple products on body composition and blood lipids: exploring the factors responsible for variability in their efficacy. Nutrients $\mathbf{9}, 746$.

118. Garcia-Conesa MT, Chambers K, Combet E, et al. (2018) Meta-analysis of the effects of foods and derived products containing ellagitannins and anthocyanins on cardiometabolic biomarkers: analysis of factors influencing variability of the individual responses. Int J Mol Sci 19, E694.

119. Martini D, Chiavaroli L, Gonzalez-Sarrias A, et al. (2019) Impact of foods and dietary supplements containing hydroxycinnamic acids on cardiometabolic biomarkers: a systematic review to explore inter-individual variability. Nutrients 11, E1805.

(1)

